\title{
Delayed diagnosis of impacted partial denture in a patient with mental retardation
}

Myeong Jong $\underline{\operatorname{Le}}^{1}$, MD, PhD

ABSTRACT Foreign body ingestion is a common problem. To minimise any complications arising from an ingested foreign body, early diagnosis and proper treatment are essential. However, this may be a diagnostic challenge, especially in patients who have psychiatric disorders or are mentally challenged. We report a case of delayed diagnosis of an impacted partial denture in the pharynx of a man with mental retardation.

Keywords: denture, dysphagia, mental retardation, microlaryngoscope

\section{INTRODUCTION}

Ingestion of a sharp foreign body can lead to gastrointestinal tract perforation, which is associated with high morbidity. Successful removal of the foreign body without surgery depends on several factors, including the location, size, shape and texture of the foreign body, the skill of the physician, and the time taken to reach a diagnosis before mucosal oedema and necrosis occur. ${ }^{(1-3)}$ We report the case of a 45-year-old intellectually challenged man who presented with sore throat, dysphagia and cough for three months. An impacted partial denture in the pharynx was discovered during subsequent clinical evaluation and extracted uneventfully. This case highlights that adequate communication and cooperation among caregivers, physicians and otolaryngologists, together with thorough clinical examination, are essential in order to achieve the best outcome for patients who are intellectually challenged.

\section{CASE REPORT}

A 45-year-old institutionalised man with mental retardation and right hemiplegia after brain surgery lost his partial denture while coughing about three months prior to his presentation to our institute. However, the patient failed to realise that he had swallowed it. Subsequently, he complained of sore throat, cough and dysphagia. Together with his caregiver, the patient visited a local clinic, but the physician did not find the partial denture. As the symptoms persisted, the patient presented to a hospital, where the otolaryngologist found the impacted partial denture in his pharynx and transferred him to the emergency department of our hospital.

Due to the patient's low intellectual status, we were unable to obtain his definitive medical history. His caregiver was only able to provide limited information, such as the fact that the patient had been taking an anticonvulsant for his intermittent seizures (several times a year) after craniectomy. At the emergency department, the patient's vital signs were stable.

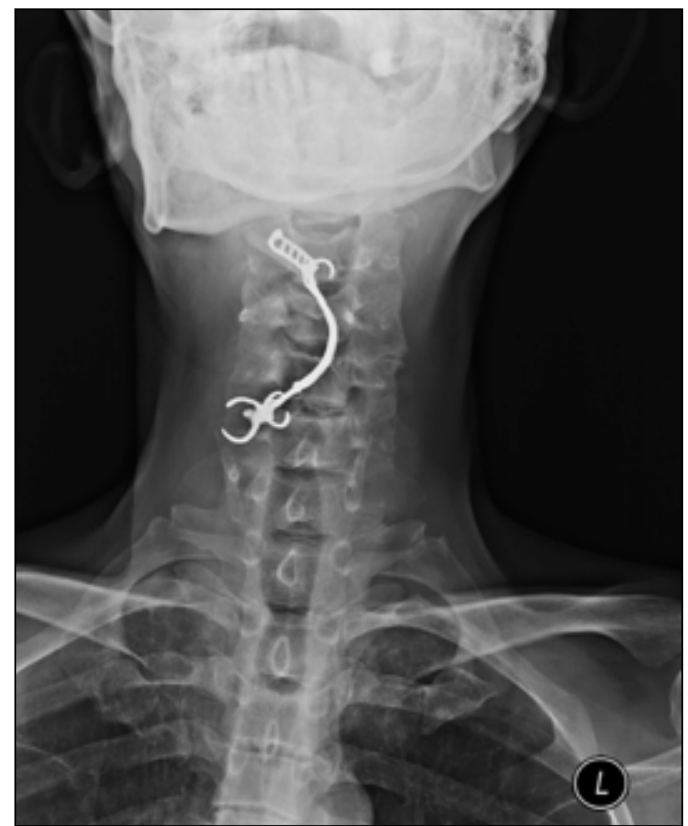

Fig. 1 Anteroposterior radiograph of the neck shows a metallic foreign body in the pharynx.

Radiographic examination was performed in order to localise the foreign body. The denture was observable by means of a neck radiograph (Fig. 1), but it was not visible on posteroanterior chest radiograph (Fig. 2). Computed tomography (CT) of the neck was also taken to estimate the extent of impaction and look for evidence of oesophageal perforation, considering the length of time the foreign body had been in situ (Fig. 3). After confirmation of the partial denture in the pharynx through rigid laryngoscopy, a gastroenterologist was consulted to determine the best course for flexible esophagoscopic removal of the denture.

During flexible esophagoscopy, the distal end of the denture was not found due to belching and coughing. It was recommended that the piece be extracted under general anaesthesia. After discussion with the surgical team, rigid

${ }^{1}$ Department of Anaesthesiology and Pain Medicine, Konkuk University Medical School, Republic of Korea

Correspondence: Dr Myeong Jong Lee, Assistant Professor, Department of Anaesthesiology and Pain Medicine, Konkuk University Medical School, Konkuk University Chungju Hospital, Kyohyun 2-dong, Chungju-si, Chungbuk-do 380-704, Republic of Korea. gooddr21@naver.com 


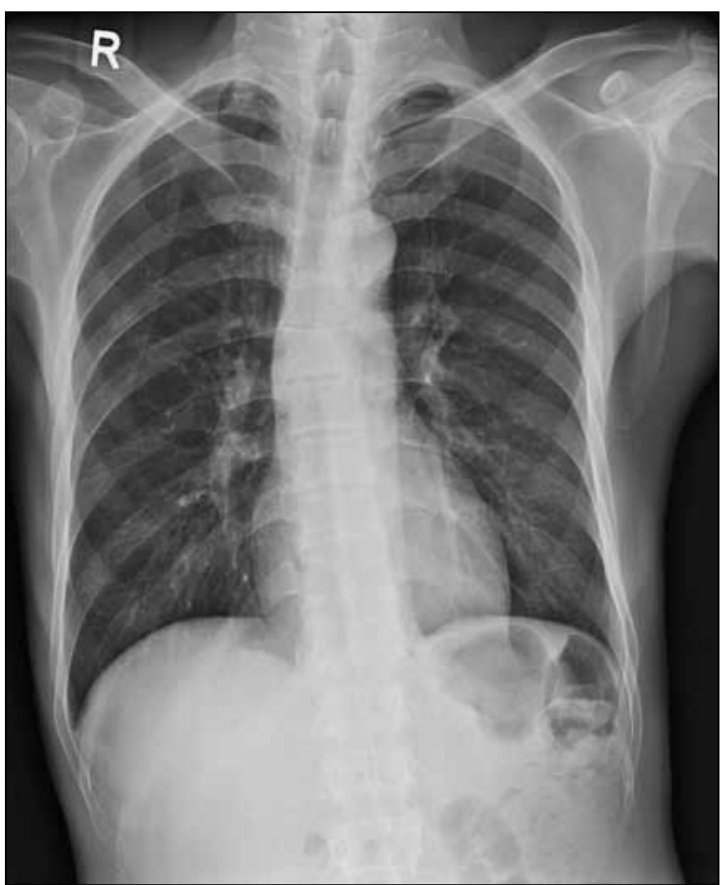

Fig. 2 Posteroanterior radiograph of the chest region shows clear fields.

microlaryngoscopic extraction before surgery was planned. However, neck CT suggested that the denture in the pharynx could be an obstacle to endotracheal intubation with direct laryngoscopy; in view of a possible difficult intubation, an awake intubation was planned. Following intravenous injection of midazolam $5 \mathrm{mg}$, an awake sedated fibreoptic intubation was performed without any difficulties, and the denture (Fig. 4) was removed with the aid of microlaryngoscopy. The patient had an uneventful recovery.

\section{DISCUSSION}

Ingestion of a foreign body is a common problem, especially among young children and patients with morbidities that limit cognition and communication, such as stroke and mental retardation. ${ }^{(4,5)}$ Although the majority of swallowed objects traverse the gut uneventfully, $10 \%-20 \%$ of these objects need to be extracted via nonsurgical procedures, and less than $1 \%$ may require surgery. ${ }^{(1,4,6)}$ Ingestion of a foreign body can lead to serious complications such as retropharyngeal and intraabdominal abscesses, oesophageal impaction and stricture, tracheo-oesophageal and enterocolonic fistulas, sepsis and massive haemorrhage. ${ }^{(7-9)}$ Early diagnosis and adequate management will prevent oedematous reaction, mucosal infection and necrosis, thereby minimising further complications. Symptoms associated with foreign body ingestion may include dysphagia, odynophagia, coughing, choking and haematemesis. ${ }^{(10)}$ However, these symptoms may be nonspecific and occasionally absent. Diagnosis of foreign body ingestion may be missed or delayed in a nonverbal person, or in our case, a mentally challenged patient, which can potentially lead to further complications, as previously described. ${ }^{(3,11)}$ In addition, unnoticed ingestion of objects of an
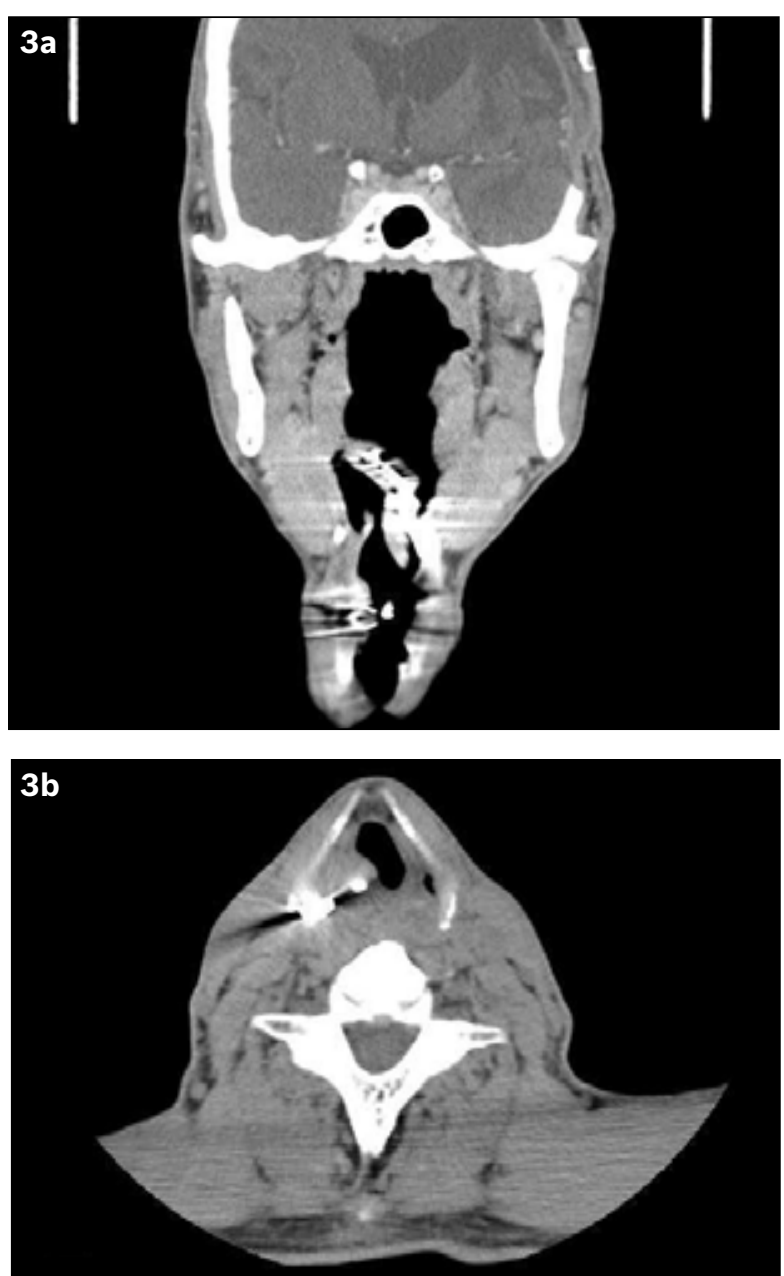

Fig. 3 CT images of the neck show a metallic foreign body in the oropharynx and hypopharynx, possibly at the opening of the (a) oesophagus and (b) deformed airway.

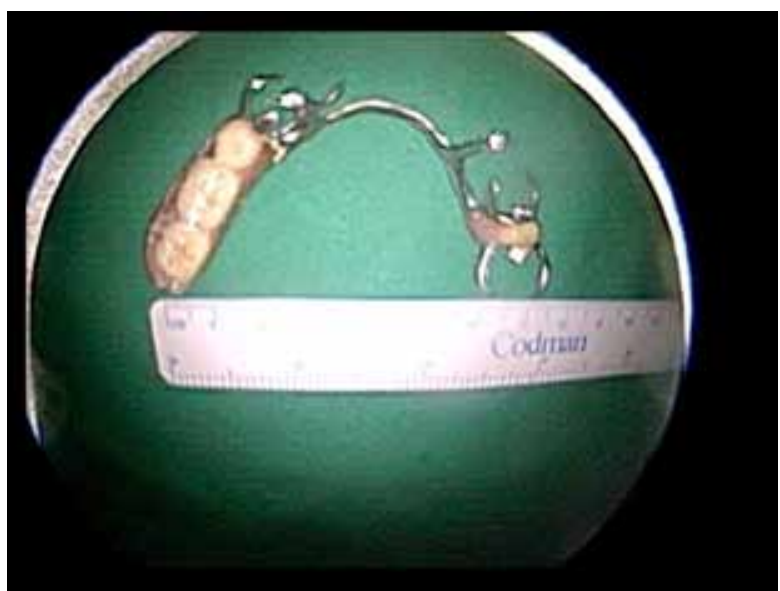

Fig. 4 Photograph shows the retrieved denture.

odontogenic origin can occur during general anaesthesia and cardiopulmonary resuscitation. ${ }^{(12,13)}$

In order to reach an early diagnosis, a cooperative, multidisciplinary approach is important. In addition to staff from the developmental centre or institution, family doctors and emergency room physicians should be highly suspicious of foreign body ingestion when a mentally challenged patient complains of medically unexplainable symptoms, including 
dysphagia, odynophagia and coughing, or refuses food. The majority of dentures appear to become impacted in the upper oesophagus and pharynx, followed by the upper thorax and lower oesophagus. ${ }^{(14)}$ Radiolucent dentures may not be visible in neck radiographs. ${ }^{(10,15)}$ Therefore, referral to an otolaryngologist should be considered in a patient with dysphagia, coughing or new-onset neck pain. Direct visualisation of the swallowed foreign body with a flexible or rigid endoscope is possible while it remains in the hypopharynx or oesophagus. Thus, an endoscopic examination should be promptly performed, as it allows for simultaneous diagnosis and removal. ${ }^{(4,10)}$

In conclusion, caregivers should be alert to the possibility of swallowed dentures among patients who cannot perceive or report the disappearance of their dentures. In addition, clinicians need to be vigilant in diagnosing foreign body ingestion among patients with persistent, medically unexplainable or sudden-onset symptoms such as odynophagia, cough and feeding difficulties.

\section{ACKNOWLEDGEMENT}

This paper was supported by Konkuk University in 2012.

\section{REFERENCES}

1. Matern $U$, Aschendorff A, Krebs A, Kohlberger E, Ruckauer KD. A new method for extracting wooden foreign bodies from the upper esophagus. Endoscopy 2000; 32:1002-3.
2. Tripathi $\mathrm{T}$, Rai $\mathrm{P}$, Singh H. Foreign body ingestion of orthodontic origin. Am J Orthod Dentofacial Orthop 2011; 139:279-83.

3. Lohiya GS, Tan-Figueroa L, Van Le H, Rusu L. Esophageal obstruction by a lemon that required esophagotomy: Thoughts on prevention. Ment Retard 2005; 43:317-21.

4. Athanassiadi K, Gerazounis M, Metaxas E, Kalantzi N. Management of esophageal foreign bodies: A retrospective review of 400 cases. Eur J Cardiothorac Surg 2002; 21:653-6.

5. Hachimi-Idrissi S, Corne L, Vandenplas Y. Management of ingested foreign bodies in childhood: Our experience and review of the literature. Eur J Emerg Med 1998; 5:319-23.

6. Tanrikulu Y, Erel S, Kismet K, et al. Accidental swallowing of partial denture: A case report. Cases J 2009; 2:9363.

7. Schwartz GF, Polsky HS. Ingested foreign bodies of the gastrointestinal tract. Am Surg 1976; 42:236-8.

8. Rodriguez-Hermosa JI, Codina-Cazador A, Sirvent JM, et al. Surgically treated perforations of the gastrointestinal tract caused by ingested foreign bodies. Colorectal Dis 2008; 10:701-7.

9. McCanse DE, Kurchin A, Hinshaw JR. Gastrointestinal foreign bodies. Am J Surg 1981; 142:335-7.

10. Hashmi S, Walter J, Smith W, Latis S. Swallowed partial dentures. J R Soc Med 2004; 97:72-5.

11. Reilly S, Carr L. Foreign body ingestion in children with severe developmental disabilities: A case study. Dysphagia 2001; 16:68-73.

12. Lau G, Kulkarni V, Roberts GK, Brock-Utne J. "Where are my teeth?" A case of unnoticed ingestion of a dislodged fixed partial denture. Anesth Analg 2009; 109:836-8.

13. Chin RY, Ellul S. Dysphagia after emergency intubation: Case report and literature review. Dysphagia 2009; 24:105-8.

14. Nwaorgu OG, Onakoya PA, Sogebi OA, Kokong DD, Dosumu OO. Esophageal impacted dentures. J Natl Med Assoc 2004; 96:1350-3.

15. Haidary A, Leider JS, Silbergleit R. Unsuspected swallowing of a partial denture. AJNR Am J Neuroradiol 2007; 28:1734-5. 